\title{
Circular lattice design for UHF geodesic dome phased array antenna with reduced footprint
}

\author{
Charles Grech ${ }^{1 *}$, Marc Anthony Azzopardi ${ }^{1}$, Victor Buttigieg ${ }^{2}$ \\ ${ }^{1}$ Faculty of Engineering, University of Malta, Malta \\ ${ }^{2}$ Faculty of Information \& Communication Technology, University of Malta, Malta \\ *email: charles@grech.space
}

\begin{abstract}
A circular planar array composed of four equilateral triangle patch antennas with a common triangular ground plane is proposed. The array is a building block for a geodesic dome phased array antenna (GDPAA) based on an icosahedron structure, primarily intended for use in the UHF amateur satellite band of 435-438 MHz. Due to the relatively long wavelength of this band, a novel design for a low-cost GDPAA with a reduced footprint is proposed. It is found that if individual elements are placed in a circular lattice, the footprint is reduced and the overall array performance and antenna isolation are improved.
\end{abstract}

Keywords - Geodesic Dome, Phased Array, Triangle Patch, Ground Station, Mutual Coupling

\section{INTRODUCTION}

The recent trend of launching multiple nanosatellites operating in a similar frequency range has given rise to a new challenge. The ground station must be capable of distinguishing one satellite from several others in close proximity and must also identify individual members of a constellation. A system capable of performing a rapid electronic scan of the sky for potential targets is required. Phased array antennas (PAAs) allow this to be achieved.

Various PAA architectures are possible [1]. The authors have observed that almost all literature is focused on phased arrays in the $\mathrm{S}, \mathrm{C}$ and $\mathrm{X}$-bands, with very few examples in the $\mathrm{P}$-band such as [2]. This paper proposes a design for a planar antenna face which may be used to construct a compact geodesic dome PAA (GDPAA) for amateur satellite tracking applications which would be especially useful in the 435$438 \mathrm{MHz}$ range in footprint-constrained applications.

\section{Planar Face Design}

Full hemispherical coverage is required for the system to retain full scanning capability in both azimuth and elevation. Similar to [3], a PAA is proposed based on triangular planar subarrays, arranged in an icosahedral geodesic dome configuration. This allows for a faceted dome antenna to be constructed which retains the advantages of a spherical PAA whilst leveraging existing planar manufacturing technology. Each polygonal face serves as a planar face (PF) in the proposed design. Every PF contains four equilateral triangle patch antennas (ETPAs) with single coaxial probe feed, which is considered sufficient to carry out $360^{\circ}$ azimuthal scanning [4]. No further subdivision of each polygonal face is proposed to keep the area to a minimum.

It has been shown [5] that arranging elements in a triangular lattice (TL) as per Fig. 1a results in a wider separation of the elements than its square lattice counterpart, due to $d_{y}$ being larger than $d_{x}$, leading to better antenna isolation. To minimize footprint, a circular lattice (CL) is hereby proposed, with one central element and three elements placed on an outer concentric ring of radius $d_{x}$ as per Fig. $1 \mathrm{~b}$.

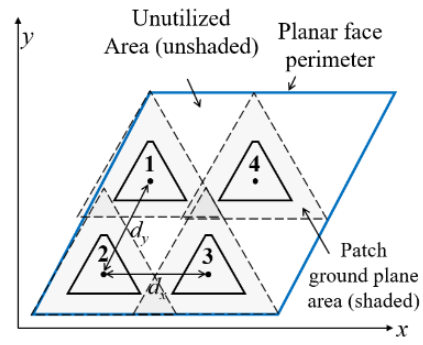

(a) Triangular Lattice

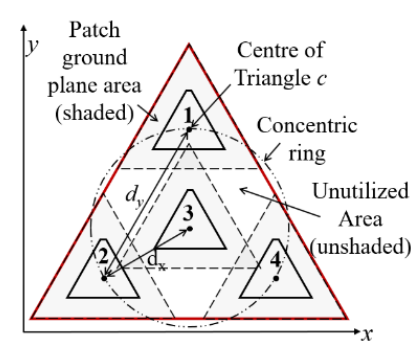

(b) Circular Lattice
Fig. 1. PF lattice options

A $1.5 \mathrm{~mm}$ aluminum common ground plane is proposed to strengthen the PF structure, simplify the manufacturing process and increase the radiation performance. In selecting the PF perimeter, where possible, each patch element has been allowed a surrounding ground plane area of similar shape with twice the area of the patch.

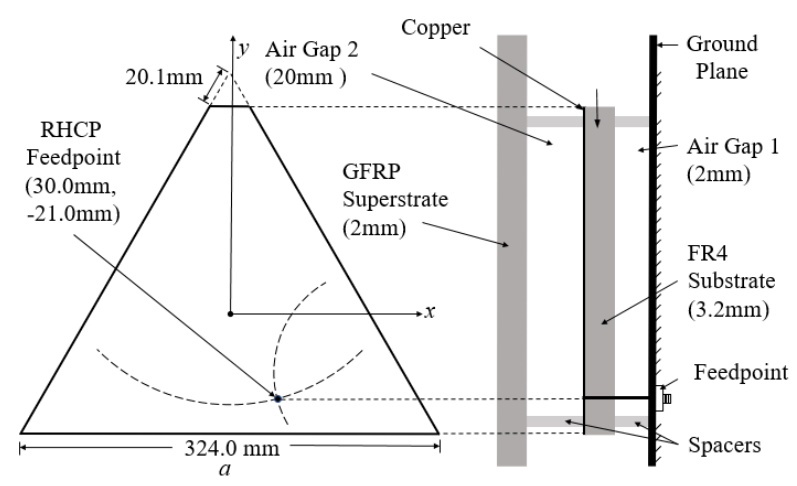

Fig. 2. Truncated-tip ETPA with single-feed

A material-based miniaturization technique has been adopted to reduce the size of the ETPA in Fig. 2 by selecting a $3.2 \mathrm{~mm}$ FR4 board as the substrate. The side length $a$ was calculated using a derivation of the simplified formula [6]: 


$$
a=2 c /\left(3 f_{r} \sqrt{ } \varepsilon_{\mathrm{r}}\right)
$$

where $f_{r}$ is the centre frequency $436.5 \mathrm{MHz}$ and $\varepsilon_{\mathrm{r}}$ is the effective permittivity of the substrate, and $c$ is the speed of light. The value of $\varepsilon_{\mathrm{r}}$ is 1.93 , calculated by a heuristic relationship taken from the capacitance of the first air gap and FR4 dielectric layers [6]. This air gap was introduced to compensate for the degradation of efficiency due to the high tangent loss property of FR4. The second air gap and glass-fibre reinforced plastic (GFRP) superstrate are introduced to serve as a protecting radome for the GDPAA. They also lower the resonant frequency and as a result $a$ is reduced further to $324.0 \mathrm{~mm}$, determined experimentally in this study.

The tip-truncation method has been selected to achieve circular polarization (CP) on ETPAs with a single feed. In this study, the concept of [7] was applied to the P-band and an optimum axial ratio (AR) was achieved with a truncation ratio (TR) of 0.062. The approximate location of the right hand circular polarized (RHCP) feed point is determined through the intersection of the $50 \Omega$ loci. These loci were calculated through field equations [8] and further fine-tuned to cater for the effects of the various dielectric layers in this study through experimentation. The value of element spacing $d_{x}=0.65 \lambda$ has been carefully selected as a compromise between footprint and mutual coupling.

\section{Planar Face Results}

The results in this section are obtained through simulations carried out using the transient solver of CST Microwave Studio. A bandwidth of $1.67 \%$ is observed for the ETPA. The AR for the ETPA is less than $6 \mathrm{~dB}$, which results in $0.45 \mathrm{~dB}$ polarization loss, considered adequate for the purpose of this study.

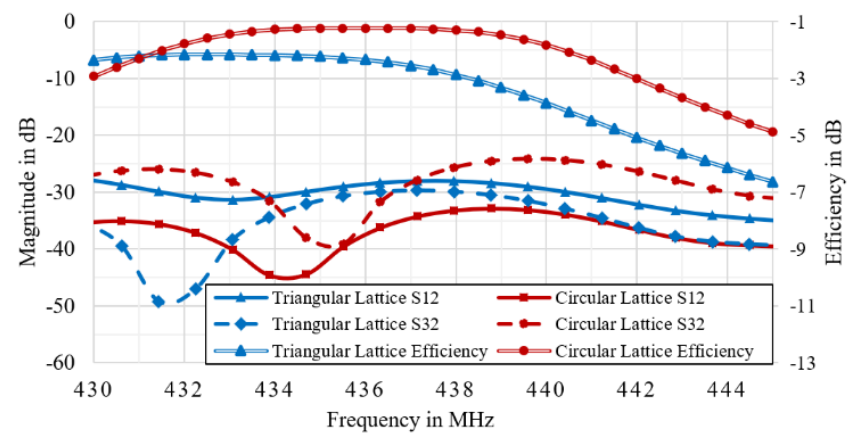

Fig. 3. Comparison of lattice mutual coupling and efficiency

In Fig. 3 the $S_{12}$ parameter is simulated on both lattices for antenna isolation comparison of elements with distance $d_{y}$ with the $S_{32}$ parameter as representation of elements which are separated with distance $d_{x}$. The antenna isolation on the proposed CL is improved. A $1 \mathrm{~dB}$ improvement in the PF efficiency is also noted resulting in a total $12.0 \mathrm{dBi}$ of realized gain for the CL combined elements. The CL design occupies circa $19 \%$ less area compared to its TL counterpart. The angular width of the CL is $34.6^{\circ}$ compared to $36.3^{\circ}$ of the TL.
This allows for better spatial resolution with the results being in agreement with [9]. An improvement in side lobe levels (SLLs) is noted without the need for any SLL reduction techniques.

\section{CONCLUSION}

Using simulation, it was shown that by placing four truncated-tip FR4 ETPAs in a CL, with one centre element and three elements on an outer concentric ring, various advantages over a TL counterpart are obtained. The patch antennas are mounted with an air gap over an aluminium ground plane together with a GFRP radome also separated with an air gap. This configuration allows for post-manufacturing tuning either via tip truncation or by adjusting air gaps. Simulations demonstrate a savings of $\sim 19 \%$ in the area of the PF which translate to a smaller GDPAA footprint. Antenna isolation and efficiency of the CF design is improved with respect to the TL counterpart. The main lobe is slightly narrower allowing for improved spatial resolution.

\section{ACKNOWLEDGEMENT}

The research work disclosed in this publication is funded by the ENDEAVOUR Scholarship Scheme (Malta). The scholarship is part-financed by the European Union - European Social Fund (ESF) under Operational Programme II Cohesion Policy 2014-2020, 'Investing in human capital to create more opportunities and promote the wellbeing of society'.

\section{REFERENCES}

[1] D. Parker, D.C. Zimmermann, "Phased arrays-part 1: theory and architectures", IEEE transactions on microwave theory and techniques, vol. 50, no.3, pp.678-687, 2002

[2] C.F. Toit, M. Deshpande, R.F. Rincon, "Advanced antenna design for NASA's EcoSAR instrument", IEEE International Symposium on Phased Array Systems and Technology (PAST), 2016, Massachusetts, United States, pp. 1-7

[3] B. Tomasic, J. Turtle, S. Liu, "The Geodesic sphere phased array antenna for satellite communication and air/space surveillance - part 1", Aerospace Corp Los Angeles CA, 2004, pp. 1-174

[4] T. Ohira, K. Gyoda, "Electronically steerable passive array radiator antennas for low-cost analog adaptive beamforming", Proceedings IEEE International Conference on Phased Array Systems and Technology, 2000, California, United States, pp. 101-104

[5] N.H. Noordin et al., "Triangular lattices for mutual coupling reduction in patch antenna arrays", Loughborough Antennas \& Propagation Conference, 2011, Loughborough, United Kingdom, pp. 1-4

[6] K.F. Lee, K.M. Luk, H.W. Lai, Microstrip Patch Antennas, World Scientific: Singapore, 2018, pp. 1-22, 189-218

[7] C.L. Tang, J.H. Lu, K.L. Wong, "Circularly polarized equilateral-triangular microstrip antenna with truncated tip", IET Electronics Letters, vol.34, no.13, pp. $1277-1277,1998$

[8] R.K. Vishwakarma, J.A. Ansari, M.K. Meshram, "Equilateral triangular microstrip antenna for circular polarization dual-band operation", Indian Journal of Radio \& Space Physics, vol. 35, no.4, pp. 293-296, 2006

[9] P. Ioannides, C.A. Balanis, "Uniform circular and rectangular arrays for adaptive beamforming applications", IEEE Antennas and Wireless Propagation Letters, Vol. 4, pp. 351-354, 2005 